\title{
Exploiting structure of maximum likelihood estimators for extreme value threshold selection
}

\author{
J. L. Wadsworth \\ Statistical Laboratory, University of Cambridge, U.K.
}

December 1, 2014

\begin{abstract}
In order to model the tail of a distribution, one has to define the threshold above or below which an extreme value model produces a suitable fit. Parameter stability plots, whereby one plots maximum likelihood estimates of supposedly threshold-independent parameters against threshold, form one of the main tools for threshold selection by practitioners, principally due to their simplicity. However, one repeated criticism of these plots is their lack of interpretability, with pointwise confidence intervals being strongly dependent across the range of thresholds. In this article, we exploit the independentincrements structure of maximum likelihood estimators in order to produce complementary plots with greater interpretability, and a suggest simple likelihood-based procedure which allows for automated threshold selection.
\end{abstract}

Keywords: diagnostic plots; extreme value modelling; maximum likelihood; threshold selection.

\section{Introduction}

When faced with the problem of making inferences on the tail of a distribution, one usually invokes the extreme value modelling paradigm. In this article we will focus on upper tails; theory for lower tails is symmetric in generality. Suppose that we are able to assume a sequence of identically distributed random variables, $\left\{X_{i} \sim F\right\}_{i=1}^{n}$, satisfying a long-range weak dependence condition (Leadbetter et al., 1983, Ch. 3), and let $x_{F}:=\inf \{x: F(x)>0\}$ and $x^{F}:=\sup \{x: F(x)<1\}$ be the lower and upper endpoints of the support. For a wide variety of $F$, the distribution of appropriately scaled excesses, $X_{i}-u$, over the threshold $u$, converges to the generalized Pareto (GP) distribution, with distribution function (d.f.)

$$
H(x)=1-[1+\xi x / \sigma]_{+}^{-1 / \xi}, \quad x>0, \quad \sigma>0, \xi \in \mathbb{R}, \quad a_{+}=\max (a, 0),
$$


as $u \rightarrow x^{F}$ (Pickands, 1975; Davison and Smith, 1990). At a practical level this leads to the approximation $\mathrm{P}\left(X_{i}>x \mid X_{i}>u\right) \approx\left[1+\xi(x-u) / \sigma_{u}\right]_{+}^{-1 / \xi}$ for a high threshold $u$, with $\xi \in \mathbb{R}$ a shape parameter and $\sigma_{u}>0$ a threshold-dependent scale parameter to be estimated; the case $\xi=0$ is to be interpreted in the limiting sense.

For a given $F$, if scaled threshold excesses follow a limiting GP distribution, then appropriately normalized maxima $M_{n}:=\max \left\{X_{1}, \ldots, X_{n}\right\}$ converge to following the generalized extreme value (GEV) distribution as $n \rightarrow \infty$, with d.f.

$$
G(x)=\exp \left\{-[1+\xi(x-\mu) / \sigma]_{+}^{-1 / \xi}\right\}, \quad \mu \in \mathbb{R}, \sigma>0, \xi \in \mathbb{R},
$$

with the shape parameter $\xi$ being the same in both $G$ and $H$. Again the GEV is treated as the approximate distribution of $M_{n}$ for a large but finite sequence length $n$. However, if all data are available then a thresholdbased modelling approach is usually preferred due to efficiency gains from an increased sample size.

These two results can be derived from a unifying point process representation due to Pickands (1971). If there exist normalization sequences $\left\{a_{n}>0\right\},\left\{b_{n} \in \mathbb{R}\right\}$ such that the sequence of point processes,

$$
\mathcal{P}_{n}=\left\{\left(\frac{i}{n+1}, \frac{X_{i}-b_{n}}{a_{n}}\right): i=1, \ldots, n\right\} \stackrel{d}{\rightarrow} \mathcal{P}, \quad n \rightarrow \infty
$$

with $\mathcal{P}$ a non-trivial limit on $(0,1) \times\left(b_{l}=\lim _{n \rightarrow \infty}\left(x_{F}-b_{n}\right) / a_{n}, \infty\right)$, then $\mathcal{P}$ is a non-homogeneous Poisson process (NHPP) with integrated intensity measure

$$
\Lambda\{(a, b) \times(x, \infty)\}=(b-a)[1+\xi(x-\mu) / \sigma]_{+}^{-1 / \xi}, \quad \mu \in \mathbb{R}, \sigma>0, \xi \in \mathbb{R}, \quad 0 \leq a<b \leq 1 .
$$

The link between the point process result and the GP and GEV distributions is explained in Coles (2001, Sect. 7.4), for example. In the convergence (1), only points for which $X_{i}$ is extreme are retained in the limit, and at a statistical level this motivates the use of the NHPP model for data exceeding a high threshold. The parameters $(\mu, \sigma, \xi)$ are respectively location, scale and shape parameters to be estimated, with $\xi$ as in $G$ and $H$. The NHPP model has the advantage over the GP model of accounting for the rate of exceedances over the threshold, and having a threshold-independent parameterization; we thus focus on this modelling strategy.

The first task in inference is to decide upon a threshold for which the approximation is adequate, taking into account the familiar bias-variance trade-off: lower thresholds induce higher bias but lower estimation variance, and vice versa. There is a growing wealth of literature devoted both to the problem of fixed threshold selection, and incorporating uncertainty in threshold selection into the inference; a recent review 

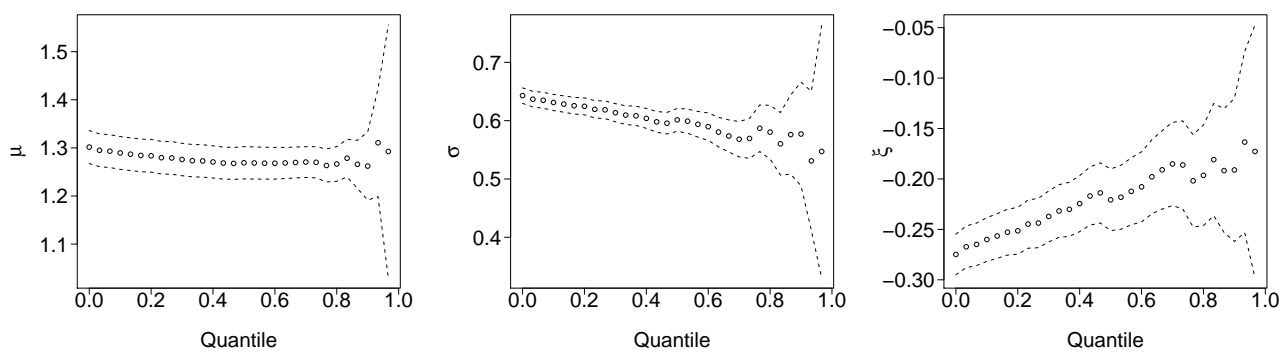

Figure 1: Traditional parameter stability plots for $(\mu, \sigma, \xi)$ in the NHPP model.

is provided by Scarrott and MacDonald (2012). In this article, we treat only the first of these issues, whilst acknowledging the importance of the latter. One of the main tools used in fixed threshold selection is the parameter stability plot: if suitable convergence has been achieved towards the limit model, then estimates of parameters $(\mu, \sigma, \xi)$ should be stable across a range of thresholds. The parameter stability plot consists of parameter estimates plotted against a range of thresholds, with pointwise confidence intervals. One then selects as a threshold the lowest value above which the parameters are deemed to be constant, attempting to take into account the uncertainty in their estimation. An example is presented in Figure 1; the difficulty in interpretation is evident. Of these three plots, those for $\sigma$ and $\xi$ tell a similar story, whilst that for $\mu$ offers little information. In the following, we thus focus mainly on plots for $\xi$; some further comments on this are given in Section 5.

In spite of the complaint that dependent confidence intervals are difficult to account for (as mentioned for instance by Scarrott and MacDonald (2012), Wadsworth and Tawn (2012) and Northrop and Coleman (2014)), the simplicity of parameter stability plots still renders their use commonplace. To produce them, one simply needs to fit the model above a range of thresholds. By contrast, to use any of the more "sophisticated" methods proposed in the literature (e.g. Beirlant et al., 1999; Ferreira et al., 2003; Wadsworth and Tawn, 2012; Northrop and Coleman, 2014, to list only a few), one needs to study more complicated methodology and / or implement bespoke code. Our aim in this article is to address the interpretability issue of parameter stability plots by exploiting the asymptotic distributional theory of the joint distribution of maximum likelihood estimators (MLEs) from overlapping samples of data, to produce diagnostics which do not require any further modelling assumptions. Furthermore, a simple testing procedure is suggested, to allow automated threshold selection if desired.

The issue of threshold selection is most commonly associated to univariate extreme value theory. However, when one wants to model the extremal dependence between two or more variables, there is typically a choice of dependence threshold as well. In the bivariate setting, an assessment of the level of dependence between two variables can be made through the coefficient of tail dependence, $\eta \in(0,1]$ (Ledford and Tawn, 1996), defined 
as follows. Suppose that random variables $\left(X_{E}, Y_{E}\right)$ have been transformed to have standard exponential margins. Then a widely-applicable regular variation assumption yields

$$
\mathrm{P}\left\{\min \left(X_{E}, Y_{E}\right)>x+t \mid \min \left(X_{E}, Y_{E}\right)>t\right\}=\frac{L\left(e^{x+t}\right)}{L\left(e^{t}\right)} \exp (-x / \eta)
$$

with $L$ slowly-varying at infinity, i.e., $\lim _{t \rightarrow \infty} L(t x) / L(t)=1$, for $x>0$. Values of $\eta>1 / 2$, and $\eta<$ $1 / 2$ indicate positive and negative extremal association respectively, whilst $\eta=1 / 2$ corresponds to near independence. The case $\eta=1$ is of special importance as this is implied by the variables being asymptotically dependent, whilst $\eta<1$ implies asymptotic independence. Different modelling strategies can be applicable to these two scenarios; see Coles (2001, Ch. 8), or Beirlant et al. (2004, Ch. 9) for further details. To estimate $\eta$, equation (2) implies an approximate exponential model for large $x>u: \mathrm{P}\left\{\min \left(X_{E}, Y_{E}\right)>x \mid\right.$ $\left.\min \left(X_{E}, Y_{E}\right)>u\right\} \approx \exp \{-(x-u) / \eta\}$. A suitable dependence threshold $u$ may again be determined through parameter stability plots for the exponential distribution inverse rate parameter $\eta$. We will also consider this problem in the sequel. Threshold selection for multivariate problems is also considered in Lee et al. (2013), but starting from an assumption of asymptotic dependence.

The remainder of the article is as follows. In Section 2 we give details of the asymptotic joint distribution of the MLEs, and use this to suggest alternative diagnostic plots. In Section 3 we outline a simple testing procedure that would allow for automated threshold selection on multiple datasets; properties and performance are assessed through simulation. Finally we consider the performance of the methods on a variety of freely-available datasets, so that the reader can compare easily with their own assessment. R code is available as supplementary material.

\section{Asymptotic joint distribution of MLEs}

\subsection{Set-up and notation}

Let us denote by $\boldsymbol{\theta} \in \Theta \subseteq \mathbb{R}^{p}$ the parameters of our model, with $\boldsymbol{\theta}_{0}$ an assumed true value. We wish to estimate $\boldsymbol{\theta}_{0}$ from data $\boldsymbol{X}$, which follow a probability model with density $f_{\boldsymbol{\theta}_{0}}$; we assume that sufficient regularity conditions for asymptotic consistency and normality of the maximum likelihood estimators hold. Suppose that the observations $X_{i}$ are mutually independent, and that each $X_{i} \in R$, for some interval or region $R$. For our purposes, $R$ can usually be considered as $\left(u_{1}, \infty\right)$, though the theory applies more generally. The joint distribution of score functions from overlapping samples is known and used in the area of sequential analysis (e.g. Siegmund (1985, p.65), or Cook and DeMets (2008, Sect. A.5)), but to our knowledge has not been exploited in this context. Since we have not found a derivation suited to our objectives, and in order 
to provide a reference on the topic, the key results will be stated here, whilst simple derivations are given as supplementary material.

If $\boldsymbol{X}$ is of random length $N$ and $(\boldsymbol{X}, N)$ represent realizations from a NHPP, with intensity $\lambda_{\boldsymbol{\theta}}(x)$ and integrated intensity $\Lambda_{\boldsymbol{\theta}}(R)=\int_{R} \lambda_{\boldsymbol{\theta}}(x) d x$, then the likelihood function on the region $R$ is

$$
L_{R}(\boldsymbol{\theta}):=f_{\boldsymbol{\theta}}(\boldsymbol{x})=\left\{\prod_{i=1}^{N} \lambda_{\boldsymbol{\theta}}\left(x_{i}\right)\right\} \exp \left\{-\Lambda_{\boldsymbol{\theta}}(R)\right\}
$$

Alternatively, if $\boldsymbol{X}$ is of fixed length $n$, consisting of independent random variables each with marginal density $f_{\boldsymbol{\theta}, 1}$, then the likelihood function is simply

$$
L_{R}(\boldsymbol{\theta})=\prod_{i=1}^{n} f_{\boldsymbol{\theta}, 1}\left(x_{i}\right)
$$

Now suppose that we partition $R$ into $R_{1}, \ldots, R_{k}$, allowing us to define several nested regions

$$
\bar{R}_{i}:=\bigcup_{j=i}^{k} R_{i}
$$

so that $R=\bar{R}_{1} \supset \bar{R}_{2} \supset \cdots \supset \bar{R}_{k}=R_{k}$. For our purposes, these regions will be $\left(u_{1}, \infty\right),\left(u_{2}, \infty\right), \ldots,\left(u_{k}, \infty\right)$, with $u_{1}<u_{2}<\cdots<u_{k}$. Denote by $\hat{\boldsymbol{\theta}}_{i}$ the MLE derived from observations on $\bar{R}_{i}=\left(u_{i}, \infty\right)$, and let $I_{i}$ be the Fisher information matrix on $\bar{R}_{i}$, with $I_{i}^{-1}$ its inverse.

\subsection{Joint distribution}

For notational convenience, we firstly state the results for a Poisson process, then detail the modifications required for likelihoods of the form (4). Since asymptotic results require that the number of points tends to infinity, but the parameters remain fixed, consider a superposition of $m$ Poisson processes. Then, with the above set-up and notation, as $m \rightarrow \infty$

$$
\left(\begin{array}{c}
m^{1 / 2}\left(\hat{\boldsymbol{\theta}}_{1}-\boldsymbol{\theta}_{0}\right) \\
m^{1 / 2}\left(\hat{\boldsymbol{\theta}}_{2}-\boldsymbol{\theta}_{0}\right) \\
\vdots \\
m^{1 / 2}\left(\hat{\boldsymbol{\theta}}_{k}-\boldsymbol{\theta}_{0}\right)
\end{array}\right) \stackrel{d}{\rightarrow} N_{p k}(\mathbf{0}, \Sigma), \quad \Sigma=\left(\left\{I^{-1}\right\}_{\min (i, j)}\right)_{1 \leq i \leq k, 1 \leq j \leq k}
$$


The $p k \times p k$ covariance matrix $\Sigma$ therefore has a block structure, with the blocks being the inverse Fisher information matrices. Here the Fisher information is

$$
I_{i}=\mathrm{E}\left\{-\nabla^{2} \log L_{\bar{R}_{i}}\left(\boldsymbol{\theta}_{0}\right)\right\}=-\Lambda_{\boldsymbol{\theta}_{0}}\left(\bar{R}_{i}\right) \mathrm{E}\left\{\nabla^{2} \log \lambda_{\boldsymbol{\theta}_{0}}(X)\right\}+\nabla^{2} \Lambda_{\boldsymbol{\theta}_{0}}\left(\bar{R}_{i}\right),
$$

the final expectation being with respect to the density $\lambda_{\boldsymbol{\theta}_{0}} / \Lambda_{\boldsymbol{\theta}_{0}}\left(\bar{R}_{i}\right)$.

The result is essentially the same for a likelihood of the form (4), where, given $m_{1}$ points on $\bar{R}_{1}$, the number of points on $\bar{R}_{i}$ is $M_{i} \sim \operatorname{Binomial}\left(m_{1}, \mathrm{P}\left(X \in \bar{R}_{i} \mid X \in \bar{R}_{1}\right)\right)$. In (5) above, replacing $m$ in the $i$ th row by $\mathrm{E}\left(M_{i}\right)=m_{1} \mathrm{P}\left(X \in \bar{R}_{i} \mid X \in \bar{R}_{1}\right)$ yields the same convergence, with

$$
I_{i}=-\mathrm{E}\left\{\nabla^{2} \log f_{\boldsymbol{\theta}_{0}, 1}(X)\right\}
$$

expectation being with respect to the density $f_{\boldsymbol{\theta}_{0}, 1}$.

\subsection{Consequences of the joint distribution}

We consider two consequences of the asymptotic distribution (5). The first of these is an independent increments structure to the MLEs, the second is a sequence of independent estimators, centered around $\boldsymbol{\theta}_{0}$. From this point onwards, distributions are assumed to hold approximately for finite $m, m_{i}$ etc., and these values appear in the variances.

\subsubsection{Independent increments}

An immediate consequence of (5) is

$$
\left(\begin{array}{c}
\left(\hat{\boldsymbol{\theta}}_{1}-\hat{\boldsymbol{\theta}}_{2}\right) \\
\left(\hat{\boldsymbol{\theta}}_{2}-\hat{\boldsymbol{\theta}}_{3}\right) \\
\vdots \\
\left(\hat{\boldsymbol{\theta}}_{k-1}-\hat{\boldsymbol{\theta}}_{k}\right)
\end{array}\right) \dot{\sim} N_{p(k-1)}\left(\mathbf{0}, \frac{1}{m} \operatorname{BlockDiag}\left(I_{i+1}^{-1}-I_{i}^{-1}\right)_{1 \leq i \leq k-1}\right)
$$

It is clear from (6) that, with estimates of the Fisher information matrices, pre-multiplication by the Cholesky factor of the covariance matrix would yield approximately independent normal random variables. In particular, if we isolate a parameter of interest, $\xi$ in our case, and denote by $m^{-1}\left\{\left(I_{i+1}^{-1}-I_{i}^{-1}\right)_{\xi, \xi}\right\}$ the asymptotic 
variance of $\hat{\xi}_{i}-\hat{\xi}_{i+1}$, then the standardized increments

$$
\left(\begin{array}{c}
\xi_{1}^{*} \\
\xi_{2}^{*} \\
\vdots \\
\xi_{k-1}^{*}
\end{array}\right):=m^{1 / 2}\left(\begin{array}{c}
\frac{\left(\hat{\xi}_{1}-\hat{\xi}_{2}\right)}{\left\{\left(I_{2}^{-1}-I_{1}^{-1}\right)_{\xi, \xi}\right\}^{1 / 2}} \\
\frac{\left(\hat{\xi}_{2}-\hat{\xi}_{3}\right)}{\left\{\left(I_{3}^{-1}-I_{2}^{-1}\right)_{\xi, \xi\}^{1 / 2}}\right.} \\
\vdots \\
\frac{\left(\hat{\xi}_{k-1}-\hat{\xi}_{k}\right)}{\left\{\left(I_{k}^{-1}-I_{k-1}^{-1}\right)_{\xi, \xi}\right\}^{1 / 2}}
\end{array}\right) \dot{\sim} N_{k-1}\left(\mathbf{0}, 1_{k-1}\right)
$$

with $1_{n}$ denoting the $n$-dimensional identity matrix. That is, asymptotically, $\boldsymbol{\xi}^{*}=\left(\xi_{1}^{*}, \ldots, \xi_{k-1}^{*}\right)^{T}$ is a sequence of independent standard normal random variables, hence we shall refer to $\boldsymbol{\xi}^{*}$ as the white noise process. This distribution is valid on an assumption that $\xi>-1 / 2$, since Smith (1985) showed that this is necessary for maximum likelihood estimators from extreme value models to behave regularly.

One often expects that MLEs from furthest into the data, $\hat{\xi}_{1}, \hat{\xi}_{2}, \ldots$, are larger or smaller than those estimated using the most extreme data, with a gradual move towards stability around a constant value from estimates derived from further into the tail. This is explained by the so-called penultimate theory of extremes, described by Gomes (1994) or Smith (1987); a brief summary of the latter is also given in Wadsworth and Tawn (2012). As a consequence, one might anticipate that departures from the null assumption of the white noise process for $\boldsymbol{\xi}^{*}$ are manifested as too many large or small values from estimates furthest into the body of the data. This is indeed often the case, and is the motivation for the likelihood-based automated selection procedure introduced in Section 3.

\subsubsection{Independent estimators of $\theta_{0}$}

A second use of (5) is to create a sequence of independent estimators that are still centered around $\boldsymbol{\theta}_{0}$. Let $i: j$ denote the sequence of indices $\{i, i+1, \ldots, j\} ; \hat{\boldsymbol{\theta}}_{i: j}$ denote the $p(j-i+1)$-vector of estimators $\left(\hat{\boldsymbol{\theta}}_{i}, \ldots, \hat{\boldsymbol{\theta}}_{j}\right)^{T} ; J_{k-j, p}$ be the $p(k-j) \times p$ matrix composed of $k-j$ vertically stacked copies of $1_{p}$, and let $\Sigma_{s, t}$ denote the submatrix of $\Sigma$ with rows and columns indexed by sequences $s$ and $t$, respectively. Then, using well-known properties of the multivariate normal, one has

$\hat{\boldsymbol{\theta}}_{j}-\Sigma_{j, j+1: k} \Sigma_{j+1: k, j+1: k}^{-1} \hat{\boldsymbol{\theta}}_{j+1: k} \dot{\sim} N_{p}\left(\boldsymbol{\theta}_{0}-\Sigma_{j, j+1: k} \Sigma_{j+1: k, j+1: k}^{-1} J_{k-j, p} \boldsymbol{\theta}_{0}, \frac{1}{m}\left(\Sigma_{j, j}-\Sigma_{j, j+1: k} \Sigma_{j+1: k, j+1: k}^{-1} \Sigma_{j+1: k, j}\right)\right)$,

and this variable is independent of $\hat{\boldsymbol{\theta}}_{j+1: k}$. Therefore, defining

$$
\tilde{\boldsymbol{\theta}}_{j}:=\left(1_{p}-\Sigma_{j, j+1: k} \Sigma_{j+1: k, j+1: k}^{-1} J_{k-j, p}\right)^{-1}\left(\hat{\boldsymbol{\theta}}_{j}-\Sigma_{j, j+1: k} \Sigma_{j+1: k, j+1: k}^{-1} \hat{\boldsymbol{\theta}}_{j+1: k}\right), \quad j=1, \ldots, k-1,
$$


and $\tilde{\boldsymbol{\theta}}_{k}=\hat{\boldsymbol{\theta}}_{k}$, the sequence of estimators $\left(\tilde{\boldsymbol{\theta}}_{1}, \ldots, \tilde{\boldsymbol{\theta}}_{k}\right)$ is asymptotically normal, centered around $\boldsymbol{\theta}_{0}$, with $\operatorname{Var}\left(\tilde{\boldsymbol{\theta}}_{j}\right)$ equal to $\left(1_{p}-\Sigma_{j, j+1: k} \Sigma_{j+1: k, j+1: k}^{-1} J_{k-j, p}\right)^{-1} \frac{1}{m}\left(\Sigma_{j, j}-\Sigma_{j, j+1: k} \Sigma_{j+1: k, j+1: k}^{-1} \Sigma_{j+1: k, j}\right)\left(1_{p}-\Sigma_{j, j+1: k} \Sigma_{j+1: k, j+1: k}^{-1} J_{k-j, p}\right)^{-T}$, and $\operatorname{Cov}\left(\tilde{\boldsymbol{\theta}}_{i}, \tilde{\boldsymbol{\theta}}_{j}\right)=0, \quad j \neq i$. This suggests a plot of the adjusted estimates of our parameter of interest, $\tilde{\xi}$, or $\tilde{\eta}$, against threshold. We include an example of such a plot in Section 3.2.3, but we have found the use of the null white noise process $\boldsymbol{\xi}^{*}$ more useful in practice, and thus we focus mainly on that.

\section{Likelihood ratio test and simulation results}

\subsection{Likelihood ratio testing for white noise}

As detailed in Section 2.3.1, the null (i.e., when the data arise from the assumed extreme value model) asymptotic distribution of the increment process $\boldsymbol{\xi}^{*}$ is a sequence of independent standard normal random variables, and due to the structure of extreme value theory problems, we expect departures from this null distribution to be more frequent from estimates derived further into the body of the distribution. That is, we expect that $\boldsymbol{\xi}_{1: j}^{*}$ is less likely to be white noise than $\boldsymbol{\xi}_{j+1: k-1}^{*}$. With this in mind, consider the following simple changepoint model for $\boldsymbol{\xi}^{*}$ :

$$
\begin{aligned}
& \xi_{i}^{*} \sim N(\beta, \gamma) \text { iid, } \quad i=1, \ldots, j, \\
& \xi_{i}^{*} \sim N(0,1) \text { iid, } \quad i=j+1, \ldots, k-1,
\end{aligned}
$$

based on a simplistic assumption that below an appropriate threshold, $\boldsymbol{\xi}_{1: j}^{*}$ might be better approximated by a $N(\beta, \gamma)$ distribution than a $N(0,1)$ distribution. Some motivation for this is provided by the fact that under model misspecification, estimators are often still asymptotically normal, but with adjusted means and covariances (White, 1982). The likelihood function associated to (7) is:

$$
L(\beta, \gamma, j)=\prod_{i=1}^{k-1} \phi\left(\xi_{i}^{*} ; \beta, \gamma\right)^{\mathbb{1}(i \leq j)} \phi\left(\xi_{i}^{*} ; 0,1\right)^{\mathbb{1}(i>j)}, \quad \beta \in \mathbb{R}, \gamma>0, j \in\{2, \ldots, k-1\}
$$

with $\phi(\cdot ; \beta, \gamma)$ the $N(\beta, \gamma)$ probability density function. To identify a threshold that provides the best fit to the likelihood (8), we maximize the profile likelihood $L_{p}(j)=L\left(\hat{\beta}_{j}, \hat{\gamma}_{j}, j\right)$, with $\left(\hat{\beta}_{j}, \hat{\gamma}_{j}\right)$ the MLEs for a fixed $j$. Let $j^{*}=\arg \max _{j} L_{p}(j)$. The question of interest is then: "does $L\left(\hat{\beta}_{j^{*}}, \hat{\gamma}_{j^{*}}, j^{*}\right)$ give a significantly better fit to $\boldsymbol{\xi}^{*}$ than $L(0,1,0)$ ?", where $L(0,1,0)=\prod_{i=1}^{k-1} \phi\left(\xi_{i}^{*} ; 0,1\right)$. This question may be answered via a 
likelihood ratio test, with test statistic

$$
T=\frac{L\left(\hat{\beta}_{j^{*}}, \hat{\gamma}_{j^{*}}, j^{*}\right)}{L(0,1,0)}
$$

The null distribution of $T$ is calculated easily by simulation. If the value of $T$ is significant at some userdefined level $\alpha$, then there is evidence against a hypothesis of white noise, and we select as a threshold that which provides the best fit to $(8), u^{*}=u_{j^{*}+1}$. (The indexing means that we associate $\xi_{j}^{*}=\left(\hat{\xi}_{j}-\right.$ $\left.\hat{\xi}_{j+1}\right) /\left\{\left(I_{j+1}^{-1}-I_{j}^{-1}\right)_{\xi, \xi}\right\}^{1 / 2}$ with the higher of the two thresholds involved.) Otherwise there is no evidence that the sequence $\boldsymbol{\xi}^{*}$ is different from white noise, and we take the lowest threshold in contention. This procedure echoes maximum likelihood changepoint estimation (Eckley et al., 2011, Section 10.2.1).

One possible criticism of this approach is that we approximate what we suppose may often be smooth change towards white noise with a simpler changepoint model. However, since the departures from white noise are generally unknown, this approach at least provides a parsimonious approximation to reality. The lowest threshold that one entertains, $u_{1}$, may also have an impact upon the selected threshold, and might thus be regarded as a tuning parameter. To counteract any effect of this, the testing procedure could be iterated if desired by re-defining $j^{*}+1=1$, but we do not consider this further here.

A natural question that arises is how many thresholds $k$ one should choose. There should be some link to the sample size of the data: if $k$ is too large compared to the sample size $n$, then the asymptotic theory will not provide a good approximation to the distribution. One way to test this is to examine the distribution of p-values associated to $T$ under the null hypothesis for a variety of $n$ and $k$; the distribution should be uniform when the approximation is adequate.

Table 1 offers some insight into reasonable ranges of $k$ and $n$, displaying approximate p-values from Kolmogorov-Smirnov tests of uniformity of the p-value distribution, when the LR test was applied under the null hypothesis. The three different sections of the table correspond to two different parameterizations of the exponential model, and the NHPP model. Interestingly, the picture is slightly different between the two exponential cases: for the inverse rate $(\eta)$ parameterization, $n$ generally needs to be larger for a given $k$ for the distributional results to be accurate. The table is intended as a guide only, as the Kolmogorov-Smirnov test produces only approximate p-values in the presence of ties, which occur here due to p-values being derived from simulated distributions. Furthermore, the algorithm used is such that $k$ may be reduced in case of numerical failure as described in Section 3.2.1. In Section 3.2 we take $k=25$ for $n=5000$, which appears adequate. In the case of measured data, which are inherently less smooth than simulated data, the choice of $k$ may give more variable results; some further comments on this are made in Section 5 .

It is also worth noting that many other tests are possible for departures from white noise; that which we 


\begin{tabular}{rrrrrrrrrrrr}
\hline & $k \mid n:$ & 1000 & 2000 & 3000 & 4000 & 5000 & 6000 & 7000 & 8000 & 9000 & 10000 \\
\hline & 10 & 0.78 & 0.80 & 0.20 & 0.98 & 0.85 & 0.55 & 0.76 & 0.97 & 0.46 & 0.24 \\
& 20 & 0.00 & 0.06 & 0.08 & 0.35 & 0.63 & 0.23 & 0.12 & 0.53 & 0.44 & 0.23 \\
(a) & 30 & 0.00 & 0.38 & 0.06 & 0.15 & 0.21 & 0.01 & 0.06 & 0.64 & 0.14 & 0.79 \\
& 40 & 0.00 & 0.00 & 0.02 & 0.05 & 0.00 & 0.03 & 0.01 & 0.28 & 0.46 & 0.95 \\
& 50 & 0.00 & 0.00 & 0.00 & 0.00 & 0.00 & 0.00 & 0.01 & 0.52 & 0.52 & 0.10 \\
\hline & 10 & 0.02 & 0.27 & 0.12 & 0.95 & 0.62 & 0.84 & 0.98 & 0.45 & 0.41 & 0.31 \\
& 20 & 0.00 & 0.00 & 0.06 & 0.02 & 0.28 & 0.01 & 0.08 & 0.78 & 0.20 & 0.12 \\
(b) & 30 & 0.00 & 0.00 & 0.00 & 0.00 & 0.00 & 0.00 & 0.03 & 0.68 & 0.10 & 0.31 \\
& 40 & 0.00 & 0.00 & 0.00 & 0.00 & 0.00 & 0.00 & 0.01 & 0.02 & 0.16 & 0.01 \\
& 50 & 0.00 & 0.00 & 0.00 & 0.00 & 0.00 & 0.00 & 0.00 & 0.08 & 0.00 & 0.00 \\
\hline & 10 & 0.00 & 0.08 & 0.16 & 0.62 & 0.88 & 0.85 & 0.15 & 0.86 & 0.29 & 0.50 \\
& 20 & 0.00 & 0.00 & 0.01 & 0.15 & 0.33 & 0.03 & 0.02 & 0.91 & 0.38 & 0.31 \\
(c) 30 & 0.00 & 0.00 & 0.00 & 0.00 & 0.03 & 0.14 & 0.00 & 0.16 & 0.56 & 0.07 \\
& 40 & 0.00 & 0.00 & 0.00 & 0.00 & 0.00 & 0.00 & 0.00 & 0.02 & 0.01 & 0.06 \\
& 50 & 0.00 & 0.00 & 0.00 & 0.00 & 0.00 & 0.00 & 0.00 & 0.00 & 0.03 & 0.00 \\
\hline
\end{tabular}

Table 1: Approximate p-values from Kolmogorov-Smirnov tests for uniformity of the p-value distribution of the LR test, under the null hypothesis, using (a) the exponential distribution with rate parameterization; (b) the exponential distribution with inverse rate parameterization $(\eta)$; (c) the NHPP model.

have detailed here is motivated by the structure of an extreme value problem, and the kind of departures we expect to see from the null distribution, but we make no claim of optimality. Nonetheless, performance in simulation studies appears adequate, and broadly in agreement with values suggested by reasonable interpretation of traditional parameter stability plots. Thus the testing procedure could be used for automated threshold selection on multiple datasets if desired.

\subsubsection{Contrast with other approaches}

Two recent approaches to improving threshold diagnostic plots and / or threshold selection rules under relatively few assumptions on the main body of the data are found in Northrop and Coleman (2014) and Lee et al. (2013). Northrop and Coleman (2014) begin with an assumption of multiple shape parameters at different thresholds, motivated by penultimate extreme value theory. Following this, they apply a score test to assess whether all shape parameters are equal, plotting p-values versus threshold: rejection of the hypothesis suggests that a higher threshold is required. They suggest two alternative methods for automating threshold selction: either taking the lowest threshold such that the associated p-value is non-significant (at some level $\alpha$ ), or the lowest threshold such that all p-values associated to that and all higher thresholds are non-significant. One drawback of this approach is that under the null hypothesis, there are approximately $\alpha \times 100 \%$ rejections at each threshold (cf. Figure 2 in Northrop and Coleman (2014)), so that in applying such selection rules under the null, more than $\alpha \times 100 \%$ of repetitions will select a threshold higher than the minimum. 
Lee et al. (2013) describe threshold diagnostic plots based on Bayesian measures of "surprise". Their plots consist of posterior predictive p-values of certain test statistics in an attempt to diagnose departures from the assumed model. In contrast to frequentist p-values, posterior predictive p-values should be close to 0.5 , and failure of the model is indicated by departures from this. However it does not seem clear how to choose a good automated selection rule whose properties can be established.

The method described here also has a subjective element, in the selection of the particular testing procedure for white noise. However, an attractive feature of the approach is that the behaviour can be easily understood under the asymptotic regime and null hypothesis: for a test of size $\alpha$, we should select the lowest threshold in contention $(1-\alpha) \times 100 \%$ of the time.

\subsection{Simulation Results}

Here we detail the performance of the likelihood ratio test suggested in Section 3 for automated threshold selection in a variety of scenarios. Aside from the univariate case, on which we have largely focussed, we will also consider the methodology for the coefficient of tail dependence, $\eta$, described in Section 1. We firstly describe the calculation of the information matrices used.

\subsubsection{Information matrices}

The simplest way to estimate the information matrix from the NHPP model is to use a numerically differentiated Hessian, readily available in many standard optimization routines. In order to find the white noise process $\boldsymbol{\xi}^{*}$, we require that the elements $\left(I_{i+1}^{-1}-I_{i}^{-1}\right)_{\xi, \xi}, i=1, \ldots, k-1$, are positive. In most situations this is the case, however when it is not, we can either change the threshold values, or change the number of thresholds $k$. Here we used fixed empirical quantiles for $\boldsymbol{u}=\left(u_{1}, \ldots, u_{k}\right)$, with associated nonexceedance probabilities equi-spaced on an interval $\left[p_{1}, p_{2}\right]$. If the covariance matrix for $\left(\hat{\xi}_{1}, \ldots, \hat{\xi}_{k}\right)^{T}$ is not positive-definite for the selected thresholds, then we reduce $k$ until it is.

A further problem arises when some values of $\left(I_{i+1}^{-1}-I_{i}^{-1}\right)_{\xi, \xi}$ are simply too small, giving rise to much larger values than one would expect to see under the null for elements of $\boldsymbol{\xi}^{*}$. This leads to far too many small p-values when data is simulated from a NHPP, which should yield a uniform distribution for the p-values. To address this issue, we replaced numerically-differentiated Hessians by the expected information matrices, calculated by numerical integration of the analytically differentiated negative log-likelihoods, and evaluated at the MLEs. We also considered the analytically differentiated observed information, but this continued to give rise to similar problems, whilst the use of expected information largely rectifies the issue.

For the bivariate case, the exponential model with rate $(\theta)$ and inverse rate $(\eta)$ parameterization have 


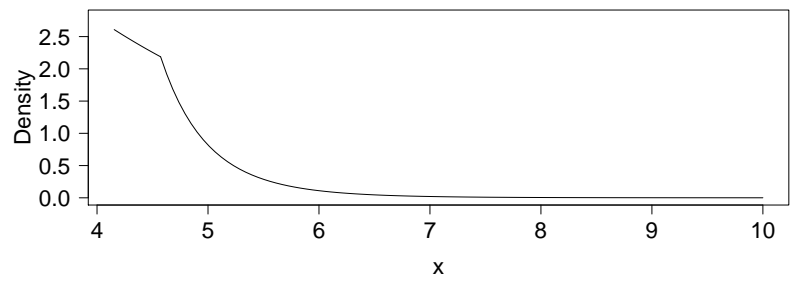

Figure 2: Density of the NHPP with changepoint at 4.57 (distribution (a)).

expected information for a single observation of $1 / \theta^{2}$ and $1 / \eta^{2}$. For $m_{i}$ observations on $\bar{R}_{i}$ we thus use total information of $m_{i} / \hat{\theta}_{i}^{2}$ or $m_{i} / \hat{\eta}_{i}^{2}$, which is also an estimate of $m_{1} \mathrm{P}\left(X \in \bar{R}_{i} \mid X \in \bar{R}_{1}\right) / \theta^{2}$ (cf. Section 2.2), and similarly for $\eta$.

\subsubsection{Univariate case: $\xi$}

We simulated 200 datasets of size / expected size 5000 according to the following distributions:

(a) NHPP on $(4.15, \infty)$ with a changepoint in intensity at 4.57 ;

(b) NHPP on $(4.27, \infty)$ with no changepoint (true parameters $\mu=10, \sigma=1, \xi=0.1$ );

(c) Truncated (at zero) standard normal (theoretical $\xi=0$ );

(d) Truncated (at zero) $t_{\nu}$-distribution, with $\nu=3$ degrees of freedom (theoretical $\xi=1 / 3$ ).

In case (a) there were an expected number of 2500 points either side of the changepoint at 4.57; parameters $(\mu, \sigma, \xi)$ changed from $(21.4,1.4,-0.05)$ below the changepoint, to $(10,1,0.1)$ above the changepoint, with the intensity constrained to be continuous at the change; the density of this is plotted in Figure 2. We apply the likelihood ratio (LR) testing procedure to the datasets to assess the quality of the inference when using this to automatically select a threshold. In each case, there were $k=25$ estimates considered ranging from the 0 to $96 \%$ quantiles. For comparison we also consider the method of Northrop and Coleman (2014) (denoted NC), taking as the selection criterion "the lowest threshold above which the p-value is larger than 0.05 and remains larger than 0.05 at all higher thresholds". If no threshold satisfied this rule then the highest threshold was used.

Table 2 details summaries of (i) the distribution of thresholds selected by the two methods, with significance level $\alpha=0.05$; (ii) the distribution and accuracy (as measured by root mean squared error, RMSE) of the estimates of $\xi$; and (iii) the accuracy of the estimates of three high quantiles, or return levels, as measured by RMSE. 
For all distributions the proposed method yields an improvement in RMSE. For case (a), the threshold at which the changepoint occurs is 4.57 , thus the mean threshold taken by the LR test, at 4.51 , is slightly too low, and leads to a small negative bias in the estimation of $\xi$. However, the range of thresholds selected is quite narrow, suggesting that the change is being identified. The mean threshold taken by the NC method is slightly too high and there is more variability in the selection, leading to worse overall performance. A similar comment applies to the thresholds selected for (b). For the LR method the lowest threshold was selected $89.5 \%$ of the time (i.e., $10.5 \%$ of the p-values were less than 0.05 ), whilst for the $\mathrm{NC}$ method the lowest threshold was selected $69 \%$ of the time. This is due to the way this procedure is calibrated, as discussed in Section 3.1.1. For (c) and (d), the LR procedure tends to select slightly higher thresholds leading to smaller bias.

\begin{tabular}{r|rrrr|rrrrrrrr}
\hline & \multicolumn{4}{|c}{ Threshold } & \multicolumn{1}{c}{ Shape Parameter } \\
\hline & Mean & Q0.05 & Q0.5 & Q0.95 & Mean & Q0.05 & Q0.5 & Q0.95 & RMSE & RL1 & RL2 & RL3 \\
\hline LR-(a) & 4.51 & 4.42 & 4.51 & 4.60 & 0.09 & 0.05 & 0.09 & 0.14 & 0.03 & 0.99 & 1.87 & 3.21 \\
NC-(a) & 4.65 & 4.38 & 4.48 & 5.81 & 0.08 & 0.02 & 0.08 & 0.14 & 0.05 & 1.52 & 3.37 & 7.00 \\
LR-(b) & 4.31 & 4.27 & 4.27 & 4.31 & 0.09 & 0.07 & 0.10 & 0.12 & 0.04 & 0.88 & 1.61 & 2.69 \\
NC-(b) & 4.54 & 4.27 & 4.27 & 5.86 & 0.10 & 0.02 & 0.10 & 0.16 & 0.05 & 1.37 & 3.10 & 6.47 \\
LR-(c) & 1.10 & 0.52 & 1.01 & 2.06 & -0.15 & -0.22 & -0.16 & -0.02 & 0.16 & 0.41 & 0.67 & 0.95 \\
NC-(c) & 0.96 & 0.41 & 0.85 & 2.00 & -0.17 & -0.24 & -0.18 & -0.06 & 0.18 & 0.46 & 0.73 & 1.02 \\
LR-(d) & 1.21 & 0.51 & 1.11 & 2.20 & 0.25 & 0.14 & 0.24 & 0.38 & 0.12 & 20.01 & 68.91 & 228.06 \\
NC-(d) & 1.26 & 0.46 & 0.94 & 3.43 & 0.23 & 0.14 & 0.21 & 0.41 & 0.14 & 22.03 & 73.52 & 234.74 \\
\hline
\end{tabular}

Table 2: Summaries of the thresholds and parameter estimates from the simulations. Q $a$ stands for the $100 a \%$ quantile; RL1-RL3 correspond respectively to the 0.9, 0.99, and 0.999 quantiles of the distribution of the maximum point in the set of observations. LR-(a) denotes the LR method used on distribution (a), etc.

Example diagnostic plots are displayed in Figure 3; each is composed of three vertically stacked plots. The uppermost of the three plots gives the values of the $\log$-likelihood ratio $2\left\{\log L\left(\hat{\beta}_{j}, \hat{\gamma}_{j}, j\right)-\log L(0,1,0)\right\} \geq 0$ versus threshold, on a quantile scale. This is maximized in $j$ at the same point as $L\left(\hat{\beta}_{j}, \hat{\gamma}_{j}, j\right)$, and the value at the maximum is equal to $2 \log T$, as defined in (9). The title of this plot displays the p-value associated to $T$. The central plot is of the white noise process, with a horizontal line at 0 , whilst the lower plot is the traditional parameter stability plot with pointwise approximate $95 \%$ CIs. The selected thresholds are displayed by vertical lines on the central and lower plots. The likelihood ratio procedure can be seen to generally select a threshold where there are too many positive / negative points in the white noise process to the left of the threshold, and much more plausible white noise values to the right of the threshold. Of course, if preferred one could simply use these plots as a more informative and more interpretable version of the existing parameter stability diagnostic plot. 

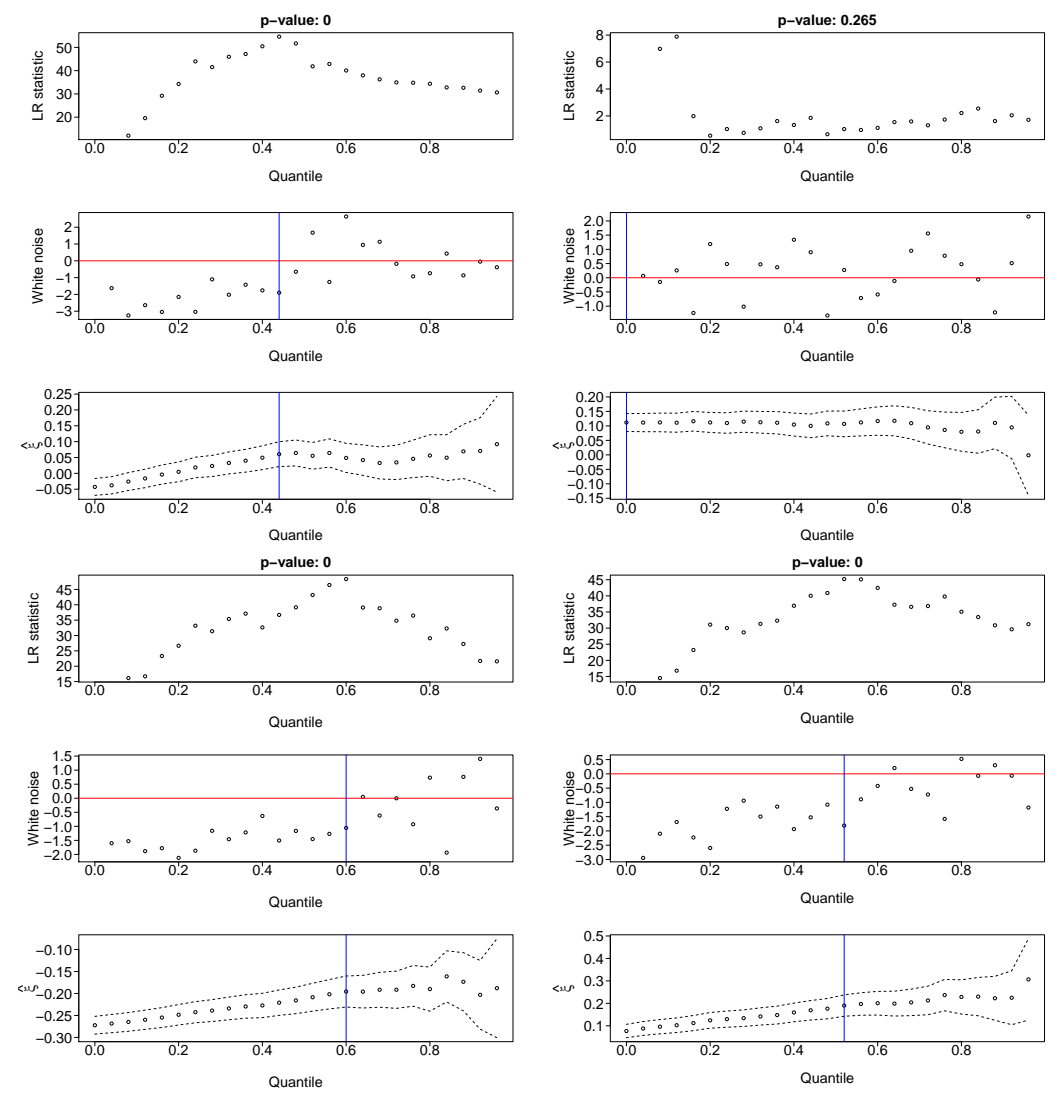

Figure 3: Example diagnostic plots for dataset (a) (top left); (b) (top right); (c) (bottom left); (d) (bottom right). The top panel in each plot shows the $2\left\{\log L\left(\hat{\beta}_{j}, \hat{\gamma}_{j}, j\right)-\log L(0,1,0)\right\}$ versus threshold; the central panel shows the white noise process $\boldsymbol{\xi}^{*}$; the bottom panel shows the traditional parameter stability plot. 


\subsubsection{Bivariate case: $\eta$}

For estimation of $\eta$, we simulated 200 datasets of size 5000 from the following dependence structures; for (b) and (c), marginal distributions were transformed to be standard exponential via the empirical probability integral transform:

(a) Independence (theoretical $\eta=0.5$ )

(b) Bivariate normal with dependence parameter $\rho=0.8$ (theoretical $\eta=0.9$ )

(c) Bivariate $t_{\nu}$-distribution, with dependence parameter $\rho=0.8$ and $\nu=2$ degrees of freedom (theoretical $\eta=1)$

Owing to the investigations in Section 3.1 that suggest the asymptotic multivariate normal distribution generally holds better for the rate parameterization of the exponential distribution, we use this for the simulation study, transforming to $\eta$ by exploiting equivariance of MLEs. Table 3 provides details of the distribution of thresholds selected by the likelihood ratio test rule with $\alpha=0.05$, along with summaries of parameter estimates, end estimates of probabilities of lying in extreme sets. These probabilities are calculated by exploiting the relation

$$
\mathrm{P}\left\{\left(X_{E}, Y_{E}\right) \in c+t+A\right\} \sim e^{-c / \eta} \mathrm{P}\left\{\left(X_{E}, Y_{E}\right) \in t+A\right\}, \quad c>0, t \rightarrow \infty
$$

for $A \subset(0, \infty)^{2}$, with addition applied componentwise. The set $A^{\prime}=t+A$ is taken to be such that $\mathrm{P}\left\{\left(X_{E}, Y_{E}\right) \in A^{\prime}\right\}$ can be estimated empirically. For case (a), $\min \left(X_{E}, Y_{E}\right)$ is exactly exponential, but for (b) and (c), the quality of the approximation depends on the rate convergence of the ratio of slowlyvarying functions in equation (2) to unity. For reference, values obtained by taking the threshold as the $90 \%$ quantile are also included. The method of Northrop and Coleman (2014) is not directly applicable here as their model assumes multiple shape parameters, whereas here it is a change in exponential rate parameter that is being sought. One could develop a procedure in the spirit of their idea for these purposes, but we do not consider this further here.

In case (a), where the data are exactly exponential, the RMSEs are smaller for the thresholds selected by the LR rule, since the test is well calibrated and hence the lowest possible threshold is usually selected (in this case $94.5 \%$ of the time); this is reflected in the quantiles of the threshold distribution. Both dependence structures (b) and (c) exhibit fairly slow rates of convergence, inducing bias in estimation of $\eta$, though the problem is worse for the bivariate normal. This bias is still present at the $90 \%$ quantile, but RMSEs are typically larger for the LR method with the extra uncertainty of the threshold incorporated. 


\begin{tabular}{rrrrrrrrrrrrr}
\hline & \multicolumn{4}{c}{ Threshold } & \multicolumn{4}{c}{ Inverse Rate Parameter } & \multicolumn{3}{c}{ Probabilities } \\
\hline & Mean & Q0.05 & Q0.5 & Q0.95 & Mean & Q0.05 & Q0.5 & Q0.95 & RMSE & p1 & p2 & p3 \\
\hline LR-(a) & 0.01 & 0.00 & 0.00 & 0.04 & 0.50 & 0.49 & 0.50 & 0.51 & 0.01 & 0.23 & 0.25 & 0.20 \\
$90 \%-(a)$ & & & & & 0.50 & 0.47 & 0.50 & 0.53 & 0.02 & 0.46 & 0.48 & 0.41 \\
LR-(b) & 0.46 & 0.05 & 0.32 & \multirow{2}{*}{1.41} & 0.81 & 0.78 & 0.81 & 0.86 & 0.09 & 0.21 & 0.33 & 1.77 \\
$90 \%-(b)$ & & & & & 0.84 & 0.80 & 0.84 & 0.88 & 0.06 & 0.24 & 0.17 & 1.57 \\
LR-(c) & \multirow{2}{*}{0.41} & \multirow{2}{*}{0.86} & \multirow{2}{*}{1.26} & \multirow{2}{*}{2.66} & 0.94 & 0.89 & 0.93 & 1.00 & 0.07 & 0.27 & 0.32 & 0.43 \\
$90 \%-(\mathrm{c})$ & & & & & 0.95 & 0.91 & 0.96 & 0.99 & 0.05 & 0.17 & 0.21 & 0.34 \\
\hline
\end{tabular}

Table 3: Summaries of the thresholds and estimates of $\eta$ from the simulations. Columns labelled p1-p3 give the MSEs of all log probabilities of the pair (in standard exponential margins) lying in extreme sets $(7,8) \times(7,8),(6.5,7.5) \times(7.5,8.5),(5.5,6.5) \times(8,9)$, respectively. LR-(a) denotes the LR method used on distribution (a), etc.
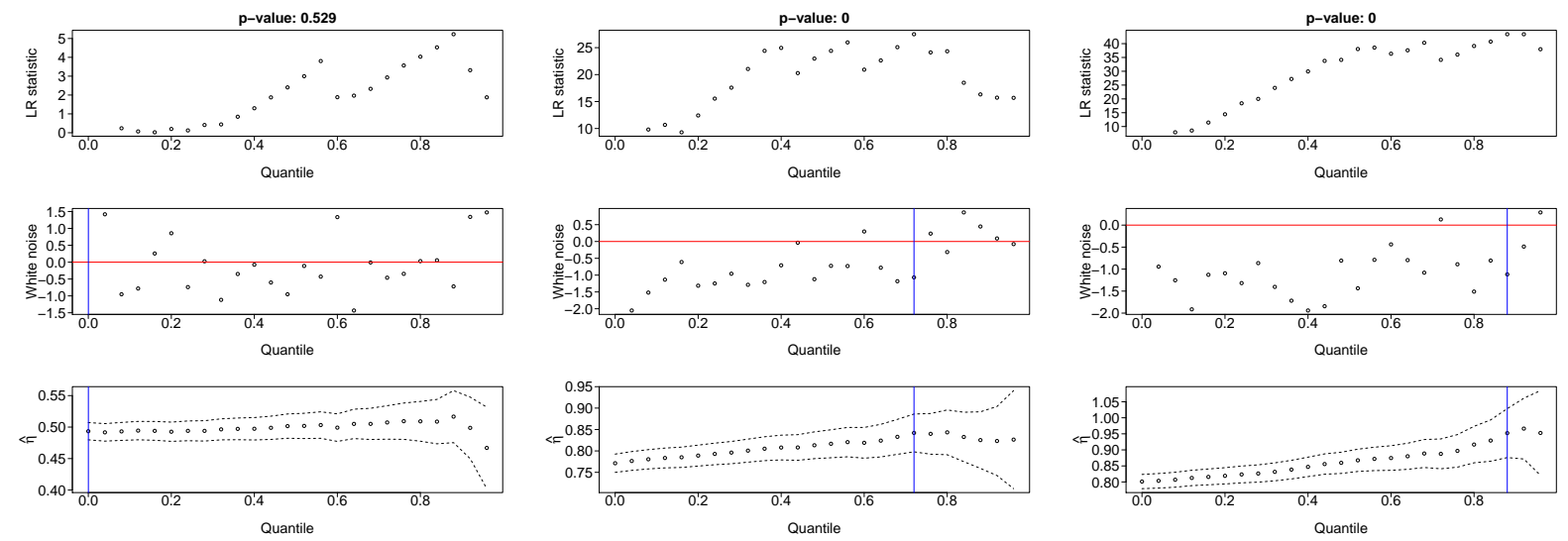

Figure 4: Diagnostic plots for the inverse rate parameter $\eta$, for dependence structures: (a) (left), (b) (centre) and (c) (right).

Example threshold diagnostic plots based on the exponential inverse rate parameter, $\eta$, are provided in Figure 4, with the same interpretation as described in Section 3.2.2 for Figure 3. Figure 5 shows an example plot based on the idea of independent estimators $\tilde{\eta}$ outlined in Section 2.3.2, for the same data as in the righthand panel of Figure 4. The confidence intervals in Figure 5 become slightly wider as the quantile decreases for two reasons: (i) the uncertainty in $\tilde{\eta}_{j}$ does not decrease much for smaller $j$, in contrast with that for $\hat{\eta}_{j}$; (ii) the confidence intervals are calibrated such that each hypothesis test of the form " $H_{0}^{(j)}: \eta_{i}=\eta_{0}$ for all $i \geq j$ " has a size of 0.05 , thus increasingly large normal quantiles are used moving from right to left. Due to the size of the confidence intervals, plots of this form do not seem to add much to the suite of threshold diagnostics, and this is why we have not pursued this line further.

\section{Applications}

Here we consider the use of the diagnostic plots from Figures 3 and 4 on some freely-available data. Code to produce these plots, written in R (R Core Team, 2014), is available online as detailed in Section 1. 


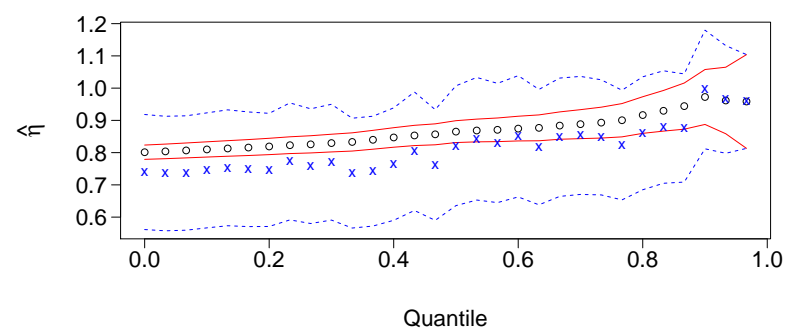

Figure 5: Alternative diagnostic plot based on the independent estimators of Section 2.3.2.

\subsection{Share Price Data}

The R package evir (Pfaff and McNeil, 2012) contains data on daily log returns of the share prices of BMW and Siemens from January 1973 until July 1996. Returns such as these typically exhibit periods of volatility, which can often be dealt with by fitting a GARCH model to the data and using the standardized residuals. For these data we applied a $\operatorname{GARCH}(1,1)$ model, as is common in the literature (e.g. Hilal et al., 2011); the resulting standardized log returns appear much closer to stationarity. The marginal diagnostic plots for the positive returns are displayed in the leftmost two panels of Figure 6. In each case the lowest threshold considered in the plot was chosen as the lowest point above which the density appeared to be decreasing, by an inspection of the histogram of the data. The number of thresholds used was $k=20$. In the case of the BMW data, the likelihood (8) for the threshold is maximized at roughly 1.47 (94\% quantile), and the evidence against white noise is significant at the $5 \%$ level. For the Siemens data, the likelihood is maximized around 1.27 (91\% quantile), and the evidence to reject a null hypothesis of white noise is even stronger.

The right-hand plot of Figure 6 displays the diagnostic for the coefficient of tail dependence between these two variables. To produce this plot, any days on which the returns are given as exactly zero for either BMW or Siemens were removed, and the remaining data were transformed to be marginally standard exponential using the empirical probability integral transform. All remaining data were used, and thus we took $k=30$ thresholds. At the selected threshold, the estimated value of $\eta$ of about 0.72 (standard error 0.01) suggests that these data are positively dependent in the extremes, but asymptotically independent.

\subsection{Fort Collins Precipitation Data}

The Fort Collins precipitation dataset, available in the R package extRemes (Gilleland and Katz, 2011), consists of daily measurements of precipitation, in inches, over a 100 year period. The data were analyzed by Katz et al. (2002); Scarrott and MacDonald (2012) also considered these data as an illustration of the difficulty of threshold selection. Although these data are available to the nearest $0.01 \mathrm{inch}$, there are many less extreme measurements which take the same value and cause the data to appear discrete in the body 

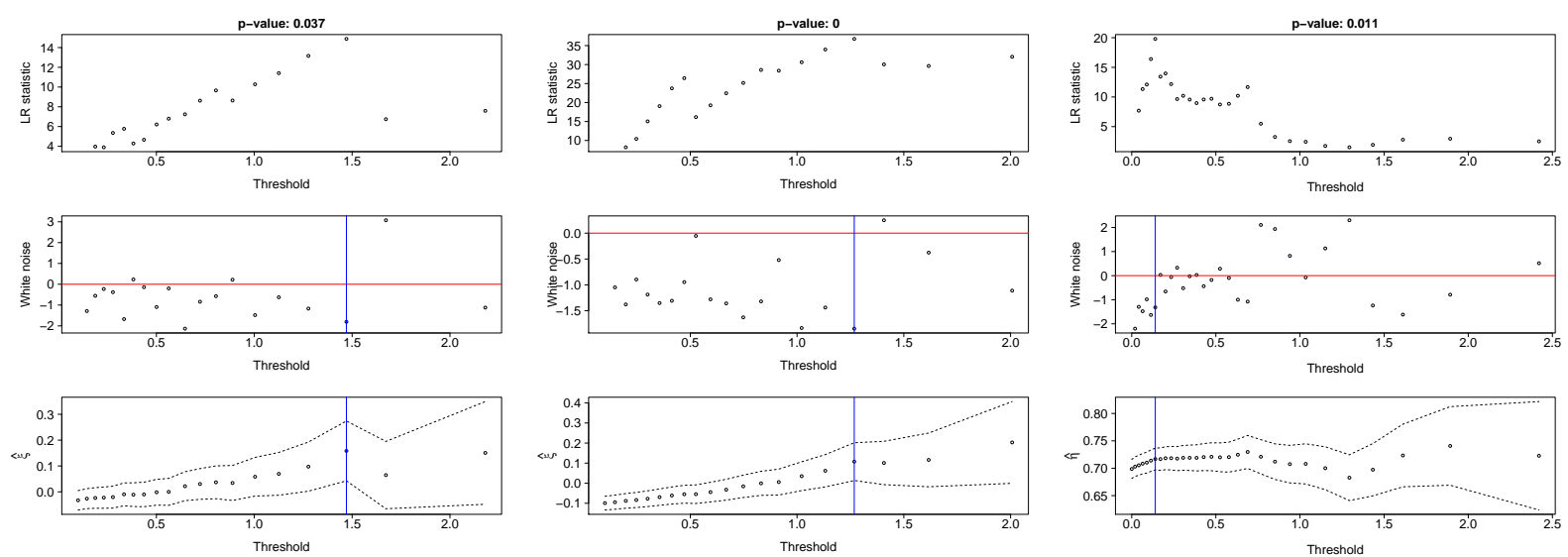

Figure 6: Marginal diagnostic plots for BMW standardized returns (left) and Siemens standardized returns (centre). Diagnostic plot for the coefficient of tail dependence (right).

and lower tail in particular. The left-hand plot in Figure 7 shows data considered above the 0.64 quantile of positive values, where there are 197 unique values out of 2916; the right-hand plot shows data above the 0.72 quantile of positive values, where there are 191 unique values out of 2171 . We used $k=20$ thresholds for the left-hand plot, and the upper 15 of those thresholds for the right-hand one. In the first case the selected threshold is 0.23 inches, lower than the 0.4 inches taken by Katz et al. (2002), but the shape parameter estimate of $\hat{\xi}=0.21$ (standard error 0.03 ) is consistent with their estimate of 0.18. In the second case, the selected threshold is 0.32 inches, and the estimate $\hat{\xi}=0.18$ (standard error 0.03 ) is consistent with the others. The likelihood (8) increases again at the highest threshold of roughly 1.2, and indeed Scarrott and MacDonald (2012) also identified 1.2 inches as a candidate threshold. We discuss repeated application of the test in Section 5 .
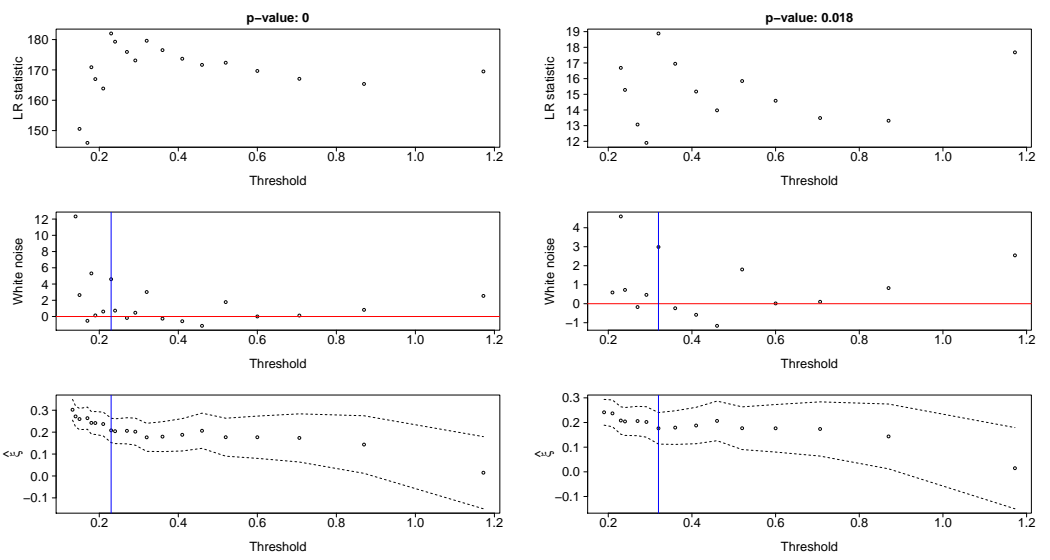

Figure 7: Threshold diagnostic plots for the Fort Collins precipitation data. 


\subsection{Loss-ALAE Data}

Data on the indemnity payments (Loss) and allocated loss adjustment expense (ALAE) relating to 1500 general liability claims from insurance companies are available in the $\mathrm{R}$ package evd (Stephenson, 2002). The data were transformed to have standard exponential margins by the empirical probability integral transform, and we concern ourselves only with the estimation of their extremal dependence structure. Figure 8 displays the diagnostic plots for dependence threshold assessment, with $k=15$ thresholds used. At the selected threshold, the MLE $\hat{\eta}=0.79$ (standard error 0.035 ), indicates moderately strong positive dependence, but asymptotic independence of the pair. However, as pointed out by Beirlant et al. (2004, p.351) the uncertainty in assessment of $\eta$ is most likely underestimated by procedures such as this one, which do not account for the uncertainty of the marginal transformations.
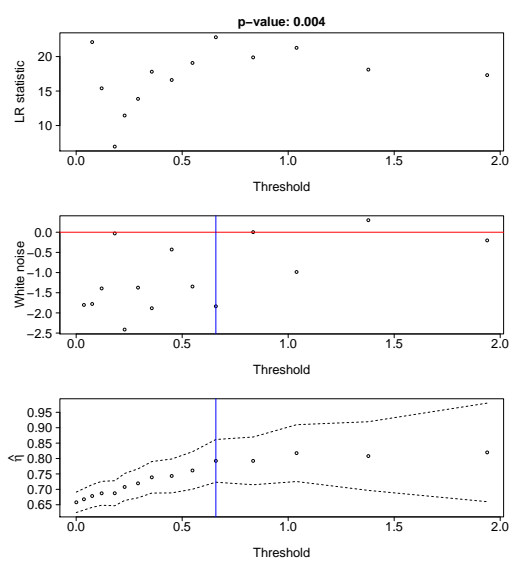

Figure 8: Dependence threshold diagnostics for the Loss-ALAE data.

\section{Discussion}

We have advocated the use of the asymptotic joint distribution of MLEs to offer a more interpretable threshold diagnostic plot. Based on the properties of this joint distribution we also suggested a likelihoodbased method for selecting a threshold, with significance assessed by simulation; performance has been assessed on both simulated data, and a variety of different measured datasets. As has been widely observed in the literature, there is rarely an obvious choice for a threshold, and methods to account for the uncertainty in threshold estimation (see e.g. MacDonald et al., 2011; Wadsworth and Tawn, 2012; Scarrott and MacDonald, 2012, and references therein), are clearly important in dealing with this. However, each such piece of methodology naturally comes with advantages and disadvantages, with none representing a panacea for the problem. As such, common applied practice remains to use fixed thresholds chosen via threshold diagnostic 
plots. The ideas proposed in this article are intended to augment this "simple" end of the threshold selection technique spectrum.

Although formulation of the white noise process $\boldsymbol{\xi}^{*}$ requires no more fits or assumptions than the formulation of a parameter stability plot, it can be the case that the estimated joint covariance matrix for $\left(\hat{\xi}_{1}, \ldots, \hat{\xi}_{k}\right)^{T}$ is not always positive definite. This has not proved to be a big problem for simulated data, but is more of an issue for measured data. One issue with environmental data in particular is rounding, such that, particularly for less extreme values, data appear discrete. The correct way to overcome this is to use a multinomial likelihood, as the true measurement could lie anywhere within the rounding limits. We have not considered this, but have noted that adding uniform noise within rounding limits tends to produce much better performance, so that such issues do seem largely attributable to the rounding. It is also noteworthy that the ideas in this paper rely on asymptotic results for the joint distribution of MLEs, and, as suggested by Table 1, may not prove useful in small samples.

We have not considered the multiple testing aspects of applying the likelihood ratio test sequentially, but one could investigate this in principle. Informally, we comment that in the Fort Collins example of Section 4.2, considering only data above 0.74 (the $95 \%$ threshold) there appears to be some evidence that a higher threshold is to be preferred, but there is some sensitivity to the number of thresholds taken. The values of the white noise process will naturally differ when different candidate thresholds are considered, and thus may sometimes suggest different selected thresholds $u^{*}$. This is a disadvantage not just of the proposed method, but fixed threshold selection in general, and often an uncomfortable feature of real data. Methods for incorporating threshold uncertainty are necessary to cope with this. The plots that we advocate here do at least help to elucidate the features of the data in this respect.

Whilst we have focussed on the white noise process for the shape parameter $\boldsymbol{\xi}^{*}$, equation (6) suggests that we could have combined information from all three parameters $(\mu, \sigma, \xi)$ across thresholds. We investigated this, but found that in general the $3 \times 3$ covariance matrix $I_{i+1}^{-1}-I_{i}^{-1}$ is rarely estimated as positive-definite. As mentioned in an asymptotic context in Section 2, one can consider the NHPP as a superposition of a number of Poisson processes, and one can in fact select a number arbitrarily for convenience. If this is chosen as, say, $N$, then the estimated parameters $(\mu, \sigma, \xi)$ correspond to the parameters for the GEV distribution for block maxima over $N$ blocks (e.g. Coles, 2001, p.133). The relationship between this tuning parameter and the number of threshold exceedances has a substantial effect on the correlations between $(\hat{\mu}, \hat{\sigma}, \hat{\xi})$ (e.g. Wadsworth et al., 2010), and may be related to this phenomenon. 


\section{Acknowledgements}

I am grateful to a referee and associate editor for constructive comments that have improved the paper.

\section{References}

Beirlant, J., Dierckx, G., Goegebeur, Y., and Matthys, G. (1999). Tail index estimation and an exponential regression model. Extremes, 2(2):177-200.

Beirlant, J., Goegebeur, Y., Segers, J., and Teugels, J. (2004). Statistics of Extremes. Wiley.

Coles, S. G. (2001). An Introduction to the Statistical Modeling of Extreme Values. Springer-Verlag, London.

Cook, T. D. and DeMets, D. L. (2008). Introduction to Statistical Methods for Clinical Trials. Chapman \& Hall / CRC.

Davison, A. C. and Smith, R. L. (1990). Models for exceedances over high thresholds (with discussion). Journal of the Royal Statistical Society. Series B (Methodological), 52(3):393-442.

Eckley, I. A., Fearnhead, P., and Killick, R. (2011). Analysis of changepoint models. In Barber, D., Cemgil, A. T., and Chiappa, S., editors, Bayesian Time Series Models. Cambridge University Press, Cambridge.

Ferreira, A., de Haan, L., and Peng, L. (2003). On optimising the estimation of high quantiles of a probability distribution. Statistics, 37(5):401-434.

Gilleland, E. and Katz, R. W. (2011). New software to analyze how extremes change over time. Eos, 92(2):13-14.

Gomes, M. I. (1994). Penultimate behaviour of the extremes. In Galambos, J., Lechner, J., and Simiu, E., editors, Extreme Value Theory and Applications, Volume 1. Kluwer Academic Publishers.

Hilal, S., Poon, S.-H., and Tawn, J. A. (2011). Hedging the black swan: Conditional heteroscedasticity and tail dependence in S\&P500 and VIX. Journal of Banking and Finance, 35:2374-2387.

Katz, R. W., Parlange, M. B., and Naveau, P. (2002). Statistics of extremes in hydrology. Advances in Water Resources, 25:1287-1304.

Leadbetter, M. R., Lindgren, G., and Rootzén, H. (1983). Extremes and Related Properties of Random Sequences and Processes. Springer Verlag, New York. 
Ledford, A. W. and Tawn, J. A. (1996). Statistics for near independence in multivariate extreme values. Biometrika, 83(1):169-187.

Lee, J., Fan, Y., and Sisson, S. A. (2013). Bayesian threshold selection for extremal models using measures of surprise. http://http://arxiv.org/abs/1311.2994.

MacDonald, A., Scarrott, C., Lee, D., Darlow, B., Reale, M., and Russell, G. (2011). A flexible extreme value mixture model. Computational Statistics and Data Analysis, 55(6):2137-2157.

Northrop, P. J. and Coleman, C. L. (2014). Improved threshold diagnostic plots for extreme value analyses. Extremes, 17(2):289-303.

Pfaff, B. and McNeil, A. (2012). evir: Extreme Values in R. R package version 1.7-3.

Pickands, J. (1971). The two-dimensional Poisson process and extremal processes. Journal of Applied Probability, 8(4):745-756.

Pickands, J. (1975). Statistical inference using extreme order statistics. Annals of Statistics, 3:119-131.

R Core Team (2014). R: A Language and Environment for Statistical Computing. R Foundation for Statistical Computing, Vienna, Austria.

Scarrott, C. and MacDonald, A. (2012). A review of extreme value threshold estimation and uncertainty quantification. Revstat, 10(1):33-60.

Siegmund, D. (1985). Sequential Analysis: Tests and Confidence Intervals. Springer-Verlag, New York.

Smith, R. (1985). Maximum likelihood estimation in a class of nonregular cases. Biometrika, 72(1):67-90.

Smith, R. L. (1987). Approximations in extreme value theory. University of North Carolina, Department of Statistics, Technical Report No. 205.

Stephenson, A. G. (2002). evd: Extreme value distributions. $R$ News, 2(2):31-32.

Wadsworth, J. L. and Tawn, J. A. (2012). Likelihood-based procedures for threshold diagnostics and uncertainty in extreme value modelling. Journal of the Royal Statistical Society: Series B, 74(3):543-567.

Wadsworth, J. L., Tawn, J. A., and Jonathan, P. (2010). Accounting for choice of measurement scale in extreme value modeling. Annals of Applied Statistics, 4(3):1558-1578.

White, H. (1982). Maximum likelihood estimation of misspecified models. Econometrica, 50(1):1-25. 


\title{
Supplementary Material to Exploiting structure of maximum
}

\author{
likelihood estimators for extreme value threshold selection
}

\author{
J. L. Wadsworth
}

Statistical Laboratory, University of Cambridge, U.K.

December 1, 2014

\section{A Derivation of joint distribution of MLEs}

\section{A.1 Poisson Process Likelihood}

Consider a Poisson process on a space $\mathcal{X}$, with intensity $\lambda_{\boldsymbol{\theta}}(x)$, and denote the integrated intensity over a region $R$, by $\Lambda_{\boldsymbol{\theta}}(R)=\int_{R} \lambda_{\boldsymbol{\theta}}(x) d x$. The likelihood function for a sample $\left(X_{1}, \ldots, X_{N}, N\right)$, with $\left(X_{1}, \ldots, X_{N}\right)$ observed on $R$, is given in (3). Define $R_{1}, \ldots, R_{k}$ and $\bar{R}_{1}, \ldots, \bar{R}_{k}$ as in Section 2. Then we can equally write the likelihood (3) as

$$
L_{R}(\boldsymbol{\theta})=\left\{\prod_{i: x_{i} \in R_{1}} \lambda_{\boldsymbol{\theta}}\left(x_{i}\right)\right\} \exp \left\{-\Lambda_{\boldsymbol{\theta}}\left(R_{1}\right)\right\} \times \cdots \times\left\{\prod_{i: x_{i} \in R_{k}} \lambda_{\boldsymbol{\theta}}\left(x_{i}\right)\right\} \exp \left\{-\Lambda_{\boldsymbol{\theta}}\left(R_{k}\right)\right\},
$$

since $\Lambda_{\boldsymbol{\theta}}(R)=\sum_{i=1}^{k} \Lambda_{\boldsymbol{\theta}}\left(R_{i}\right)$. The array of likelihoods for $\boldsymbol{\theta}$ on $\bar{R}_{1}, \ldots, \bar{R}_{k}$ is

$$
\begin{aligned}
& L_{\bar{R}_{1}}(\boldsymbol{\theta})=\left\{\prod_{i: x_{i} \in R_{1}} \lambda_{\boldsymbol{\theta}}\left(x_{i}\right)\right\} e^{-\Lambda_{\boldsymbol{\theta}}\left(R_{1}\right)} \times\left\{\prod_{i: x_{i} \in R_{2}} \lambda_{\boldsymbol{\theta}}\left(x_{i}\right)\right\} e^{-\Lambda_{\boldsymbol{\theta}}\left(R_{2}\right)} \times \cdots \times\left\{\prod_{i: x_{i} \in R_{k}} \lambda_{\boldsymbol{\theta}}\left(x_{i}\right)\right\} e^{-\Lambda_{\boldsymbol{\theta}}\left(R_{k}\right)} \\
& L_{\bar{R}_{2}}(\boldsymbol{\theta})= \\
& \left\{\prod_{i: x_{i} \in R_{2}} \lambda_{\boldsymbol{\theta}}\left(x_{i}\right)\right\} e^{-\Lambda_{\boldsymbol{\theta}}\left(R_{2}\right)} \times \cdots \times\left\{\prod_{i: x_{i} \in R_{k}} \lambda_{\boldsymbol{\theta}}\left(x_{i}\right)\right\} e^{-\Lambda_{\boldsymbol{\theta}}\left(R_{k}\right)} \\
& \begin{array}{lll}
\cdots & \cdots & \ldots
\end{array} \\
& L_{\bar{R}_{k}}(\boldsymbol{\theta})= \\
& \left\{\prod_{i: x_{i} \in R_{k}} \lambda_{\boldsymbol{\theta}}\left(x_{i}\right)\right\} e^{-\Lambda_{\boldsymbol{\theta}}\left(R_{k}\right)}
\end{aligned}
$$

To consider asymptotic behaviour, we suppose that the data arise from $m$ superpositions of such Poisson processes. Let $\hat{\boldsymbol{\theta}}_{1}, \ldots, \hat{\boldsymbol{\theta}}_{k}$ be the MLEs from $L_{\bar{R}_{1}}, \ldots, L_{\bar{R}_{k}}$, respectively. Denoting the log-likelihoods by $\ell_{\bar{R}_{1}}, \ldots, \ell_{\bar{R}_{k}}$, which we now suppose to be functions of the random data, we can write

$$
m^{1 / 2}\left(\hat{\boldsymbol{\theta}}_{j}-\boldsymbol{\theta}_{0}\right)=\left\{-\nabla^{2} \ell_{\bar{R}_{j}}\left(\hat{\boldsymbol{\theta}}_{j}\right) / m\right\}^{-1} m^{-1 / 2} \nabla \ell_{\bar{R}_{j}}\left(\boldsymbol{\theta}_{0}\right)+o_{p}(1)
$$


as $m \rightarrow \infty$, giving the usual asymptotic normal distribution, $N\left(\mathbf{0}, I_{j}^{-1}\right)$, for $j=1, \ldots, k$, and $I_{j}:=\mathrm{E}\left\{-\nabla^{2} \ell_{\bar{R}_{j}}\left(\boldsymbol{\theta}_{0}\right)\right\}$, expectation being both over the number of points on $\bar{R}_{j}$ for a single Poisson process, and their locations on $\bar{R}_{j}$. By the properties of the Poisson process, the data $X_{i}$ and numbers $N_{j}$ on each region are mutually independent, and from the above, we observe that we can write each $\nabla \ell_{\bar{R}_{j}}\left(\boldsymbol{\theta}_{0}\right)$ as a sum of independent contributions

$$
\nabla \ell_{\bar{R}_{j}}\left(\boldsymbol{\theta}_{0}\right)=\sum_{i=j}^{k} \nabla \ell_{R_{i}}\left(\boldsymbol{\theta}_{0}\right)
$$

Using the fact that $\left\{-\nabla^{2} \ell_{\bar{R}_{j}}\left(\hat{\boldsymbol{\theta}}_{j}\right) / m\right\}^{-1}=\mathrm{E}\left\{-\nabla^{2} \ell_{\bar{R}_{j}}\left(\boldsymbol{\theta}_{0}\right)\right\}^{-1}+o_{p}(1)$, we have

$$
\begin{gathered}
m^{1 / 2}\left(\hat{\boldsymbol{\theta}}_{1}-\boldsymbol{\theta}_{0}\right)=I_{1}^{-1} m^{-1 / 2} \sum_{i=1}^{k} \nabla \ell_{R_{i}}\left(\boldsymbol{\theta}_{0}\right)+o_{p}(1) \\
m^{1 / 2}\left(\hat{\boldsymbol{\theta}}_{2}-\boldsymbol{\theta}_{0}\right)=I_{2}^{-1} m^{-1 / 2} \sum_{i=2}^{k} \nabla \ell_{R_{i}}\left(\boldsymbol{\theta}_{0}\right)+o_{p}(1) \\
\ldots \\
m^{1 / 2}\left(\hat{\boldsymbol{\theta}}_{k}-\boldsymbol{\theta}_{0}\right)=I_{k}^{-1} m^{-1 / 2} \nabla \ell_{R_{k}}\left(\boldsymbol{\theta}_{0}\right)+o_{p}(1) .
\end{gathered}
$$

As $m \rightarrow \infty$, the above array converges in distribution to

$$
\left(\begin{array}{c}
\boldsymbol{T}_{1} \\
\boldsymbol{T}_{2} \\
\vdots \\
\boldsymbol{T}_{k}
\end{array}\right)=\left(\begin{array}{cccccccc}
I_{1}^{-1} & \left\{\boldsymbol{Z}_{1}\right. & + & \boldsymbol{Z}_{2} & + & \cdots & + & \left.\boldsymbol{Z}_{k}\right\} \\
I_{2}^{-1} & & & \left\{\boldsymbol{Z}_{2}\right. & + & \cdots & + & \left.\boldsymbol{Z}_{k}\right\} \\
\vdots & & & & & \ddots & & \vdots \\
I_{k}^{-1} & & & & & & \\
\boldsymbol{Z}_{k}
\end{array}\right)=\operatorname{Diag}_{1 \leq i \leq k}\left(I_{i}^{-1}\right)\left(\begin{array}{cccc}
1_{p} & 1_{p} & \cdots & 1_{p} \\
0 & 1_{p} & \cdots & 1_{p} \\
\vdots & & \ddots & \vdots \\
0 & 0 & \cdots & 1_{p}
\end{array}\right)\left(\begin{array}{c}
\boldsymbol{Z}_{1} \\
\boldsymbol{Z}_{2} \\
\vdots \\
\boldsymbol{Z}_{k}
\end{array}\right)
$$

with $1_{p}$ the $p$-dimensional identity matrix, and $\boldsymbol{Z}_{j} \sim N\left(\mathbf{0}, I_{R_{j}}\right)$ independent for $j=1, \ldots, k$. Noting that

$$
\operatorname{Cov}\left\{\left(\begin{array}{cccc}
1_{p} & 1_{p} & \cdots & 1_{p} \\
0 & 1_{p} & \cdots & 1_{p} \\
\vdots & & \ddots & \vdots \\
0 & 0 & \cdots & 1_{p}
\end{array}\right)\left(\begin{array}{c}
\boldsymbol{Z}_{1} \\
\boldsymbol{Z}_{2} \\
\vdots \\
\boldsymbol{Z}_{k}
\end{array}\right)\right\}=\left\{I_{\max (i, j)}\right\}_{1 \leq i \leq k, 1 \leq j \leq k}
$$

one can easily verify that the covariance matrix of $\left(\boldsymbol{T}_{1}, \ldots, \boldsymbol{T}_{k}\right)^{T}$ is equal to $\left(\left\{I^{-1}\right\}_{\min (i, j)}\right)_{1 \leq i \leq k, 1 \leq j \leq k}$.

\section{A.2 Likelihood with nonrandom number of points}

We now suppose that there is a nonrandom total of $m_{1}$ observations on $\bar{R}_{1}$, and without loss of generality, $\mathrm{P}(X \in$ $\left.\bar{R}_{1}\right)=1$. Let $f(x ; \boldsymbol{\theta})$ be the density of a single observation on $\bar{R}_{1}$, and $f_{\bar{R}_{j}}=f / \mathrm{P}\left(X \in \bar{R}_{j}\right), \mathrm{P}_{\bar{R}_{j}}(X \in \cdot)=\mathrm{P}(X \in$ 
·) $/ \mathrm{P}\left(X \in \bar{R}_{j}\right)$ etc., be the density and probability measure normalized over $\bar{R}_{j}$. Dependence of the probability measure on $\boldsymbol{\theta}$ is made implicit. We consider likelihoods of the form

$$
L_{\bar{R}_{j}}(\boldsymbol{\theta})=\prod_{i=1}^{m_{1}} f_{\bar{R}_{j}}\left(x_{i} ; \boldsymbol{\theta}\right)^{\mathbb{1}\left(X_{i} \in \bar{R}_{j}\right)}=\prod_{i: x_{i} \in \bar{R}_{j}} f_{\bar{R}_{j}}\left(x_{i} ; \boldsymbol{\theta}\right) .
$$

As in Section A.1, let $\ell_{\bar{R}_{j}}(\boldsymbol{\theta})=\log L_{\bar{R}_{j}}(\boldsymbol{\theta})$, and let $\ell_{\bar{R}_{j}}^{(1)}(\boldsymbol{\theta})$ be a single component of the sum. In Section A.1, we used $k$-dimensional arrays for exposition. However, we could have noted that the concatenated vector of scores

$$
\left(\nabla \ell_{\bar{R}_{1}}\left(\boldsymbol{\theta}_{0}\right), \ldots, \nabla \ell_{\bar{R}_{k}}\left(\boldsymbol{\theta}_{0}\right)\right)^{T}
$$

is a sum of mean-0 iid random vectors, and thus as long as the covariance matrix of the iid components of the sum, $\operatorname{Cov}\left\{\left(\nabla \ell_{\bar{R}_{1}}^{(1)}\left(\boldsymbol{\theta}_{0}\right), \ldots, \nabla \ell_{\bar{R}_{k}}^{(1)}\left(\boldsymbol{\theta}_{0}\right)\right)^{T}\right\}=\Sigma$ has finite entries then a multivariate normal limit holds when (A.1) is multiplied by $m_{1}^{-1 / 2}, m_{1} \rightarrow \infty$. The entries of the covariance matrix can be established by pairwise arguments, which is what we focus on here.

Consider two regions, say $\bar{R}_{j}, \bar{R}_{j+1}$, so that $\bar{R}_{j}=R_{j} \cup \bar{R}_{j+1}$. Their indexing need not be consecutive, but will reduce clutter in notation. Then

$$
\mathbb{1}\left(X_{i} \in R_{j}\right) \sim \operatorname{Bernoulli}\left(\mathrm{P}\left(X \in R_{j}\right)\right), \quad \mathbb{1}\left(X_{i} \in \bar{R}_{j+1}\right) \sim \operatorname{Bernoulli}\left(\mathrm{P}\left(X \in \bar{R}_{j+1}\right)\right) .
$$

The likelihood for a single point on $\bar{R}_{j}$ can be written

$L_{\bar{R}_{j}}^{(1)}(\boldsymbol{\theta})=\left\{\frac{f_{\bar{R}_{j}}\left(x_{i} ; \boldsymbol{\theta}\right)}{\mathrm{P}_{\bar{R}_{j}}\left(X \in R_{j}\right)}\right\}^{\mathbb{1}\left(X_{i} \in R_{j}\right)}\left\{\frac{f_{\bar{R}_{j}}\left(x_{i} ; \boldsymbol{\theta}\right)}{\mathrm{P}_{\bar{R}_{j}}\left(X \in \bar{R}_{j+1}\right)}\right\}^{\mathbb{1}\left(X_{i} \in \bar{R}_{j+1}\right)} \mathrm{P}_{\bar{R}_{j}}\left(X \in R_{j}\right)^{\mathbb{1}\left(X_{i} \in R_{j}\right)} \mathrm{P}_{\bar{R}_{j}}\left(X \in \bar{R}_{j+1}\right)^{\mathbb{1}\left(X_{i} \in \bar{R}_{j+1}\right)}$,

and on $\bar{R}_{j+1}$

$$
L_{\bar{R}_{j+1}}^{(1)}(\boldsymbol{\theta})=f_{\bar{R}_{j+1}}\left(x_{i} ; \boldsymbol{\theta}\right)^{\mathbb{1}\left(X_{i} \in \bar{R}_{j+1}\right)}=\left\{\frac{f_{\bar{R}_{j}}\left(x_{i} ; \boldsymbol{\theta}\right)}{\mathrm{P}_{\bar{R}_{j}}\left(X \in \bar{R}_{j+1}\right)}\right\}^{\mathbb{1}\left(X_{i} \in \bar{R}_{j+1}\right)}
$$

The score function for a single point on $\bar{R}_{j}$ is

$$
\nabla \ell_{\bar{R}_{j}}^{(1)}(\boldsymbol{\theta})=\nabla \ell_{R_{j}}^{(1)}(\boldsymbol{\theta})+\nabla \ell_{\bar{R}_{j+1}}^{(1)}(\boldsymbol{\theta})+\mathbb{1}\left(X_{i} \in R_{j}\right) \frac{\nabla \mathrm{P}_{\bar{R}_{j}}\left(X \in R_{j}\right)}{\mathrm{P}_{\bar{R}_{j}}\left(X \in R_{j}\right)}+\mathbb{1}\left(X_{i} \in \bar{R}_{j+1}\right) \frac{\nabla \mathrm{P}_{\bar{R}_{j}}\left(X \in \bar{R}_{j+1}\right)}{\mathrm{P}_{\bar{R}_{j}}\left(X \in \bar{R}_{j+1}\right)}
$$

with $\nabla \ell_{R_{j}}^{(1)}(\boldsymbol{\theta})=\mathbb{1}\left(X_{i} \in R_{j}\right) \nabla f_{R_{j}}(X ; \boldsymbol{\theta}) / f_{R_{j}}(X ; \boldsymbol{\theta})$. Noting that $\mathrm{E}\left\{\nabla \ell_{R_{j}}^{(1)}\left(\boldsymbol{\theta}_{0}\right)\right\}=\mathrm{E}\left\{\nabla \ell_{\bar{R}_{j+1}}^{(1)}\left(\boldsymbol{\theta}_{0}\right)\right\}=0$, and using 
conditional expectation,

$$
\operatorname{Cov}\left\{\nabla \ell_{\bar{R}_{j+1}}^{(1)}\left(\boldsymbol{\theta}_{0}\right), \mathbb{1}\left(X_{i} \in \bar{R}_{j+1}\right)\right\}=\operatorname{Cov}\left\{\nabla \ell_{\bar{R}_{j+1}}^{(1)}\left(\boldsymbol{\theta}_{0}\right), \mathbb{1}\left(X_{i} \in R_{j}\right)\right\}=\operatorname{Cov}\left\{\nabla \ell_{\bar{R}_{j+1}}^{(1)}\left(\boldsymbol{\theta}_{0}\right), \nabla \ell_{R_{j}}^{(1)}\left(\boldsymbol{\theta}_{0}\right)\right\}=0
$$

so that $\nabla \ell_{\bar{R}_{j+1}}^{(1)}\left(\boldsymbol{\theta}_{0}\right)$ is uncorrelated with all components of $\nabla \ell_{\bar{R}_{j}}^{(1)}\left(\boldsymbol{\theta}_{0}\right)$ except itself; i.e. $\operatorname{Cov}\left\{\nabla \ell_{\bar{R}_{j+1}}^{(1)}\left(\boldsymbol{\theta}_{0}\right), \nabla \ell_{\bar{R}_{j}}^{(1)}\left(\boldsymbol{\theta}_{0}\right)\right\}$ $=\operatorname{Var}\left\{\nabla \ell_{\bar{R}_{j+1}}^{(1)}\left(\boldsymbol{\theta}_{0}\right)\right\}=\mathrm{P}\left(X \in \bar{R}_{j}\right) I_{j}$, with $I_{j}=\mathrm{E}\left\{-\nabla^{2} \log f_{\bar{R}_{j}}\left(X ; \boldsymbol{\theta}_{0}\right)\right\}$.

By extension to any pair of indices, $\operatorname{Cov}\left\{\nabla \ell_{\bar{R}_{i}}^{(1)}\left(\boldsymbol{\theta}_{0}\right), \nabla \ell_{\bar{R}_{j}}^{(1)}\left(\boldsymbol{\theta}_{0}\right)\right\}=\operatorname{Var}\left\{\nabla \ell_{\bar{R}_{\max (i, j)}^{(1)}}\left(\boldsymbol{\theta}_{0}\right)\right\}$, so that normalizing the true scores by the square root of the expected number of points on the appropriate region gives the central limit convergence

$$
\left(\frac{\nabla \ell_{\bar{R}_{1}}\left(\boldsymbol{\theta}_{0}\right)}{m_{1}^{1 / 2} \mathrm{P}\left(X \in \bar{R}_{1}\right)^{1 / 2}}, \ldots, \frac{\nabla \ell_{\bar{R}_{k}}\left(\boldsymbol{\theta}_{0}\right)}{m_{1}^{1 / 2} \mathrm{P}\left(X \in \bar{R}_{k}\right)^{1 / 2}}\right)^{T} \stackrel{d}{\rightarrow} N_{p k}(\mathbf{0}, \Omega), \quad m_{1} \rightarrow \infty
$$

with $\Omega=\left\{I_{\max (i, j)}\right\}_{1 \leq i \leq k, 1 \leq j \leq k}$. The covariance matrix of the MLEs as opposed to the score follows as in Section A.1.

In practice one may select $\bar{R}_{1}, \ldots, \bar{R}_{k}$ in order to fix a certain number of points $m_{1}, \ldots, m_{k}$ in each. In this case the regions, rather than the number of points falling in each region, become random. At a practical level, with only a single sample, this does not alter the exploitation of the above results. At a theoretical level, expectations would change from being over random numbers to random regions. 